\title{
Overview of Robust Stability and Performance Methods of Systems with Structured Mixed Perturbations
}

\author{
M. Dahleh* \\ Mechanical and Environmental Engineering \\ University of California \\ Santa Barbara, CA 93106 \\ USA
}

\author{
J. C. Doyle \\ Electrical Engineering, 116-81 \\ Caltech \\ Pasadena, CA 91125 \\ USA
}

\begin{abstract}
In this paper we will outline the recently developed robust stability and performance analysis results for systems in the presence of structured mixed perturbations. To keep the presentation simple we will restrict attention to scalar perturbations. The goal of this outline is to develop in a succinct fashion an overall description of the state of the art techniques in analyzing systems with mixed perturbations, and to point the reader to sources in the literature where more details and proofs can be found. Also, the references are not intended to be a complete literature survey, but rather as a source for a more complete bibliography.
\end{abstract}

\section{Introduction}

The purpose of this overview is to present in a unified and simple manner the different approaches that have recently been developed for modeling and analyzing uncertain control systems. We will keep the presentation simple and limit ourselves in many cases to the presentation of the more basic results, and thus avoid the technical difficulties that may arise from a more general development. References and comments are given to direct the interested reader to the original sources, and to more extensive articles.

One of the most important reasons for employing feedback control is to counter the effect of uncertainty. In this outline we will consider dif-

\footnotetext{
*Research Supported by NSF Grant ECS-9111058
}

ferent types of norm-bounded perturbations, as well as real parametric uncertainties. This paper is organized as follows: in section 2 we present a mathematical description of appropriate classes of systems and perturbations, in section 3 we present robust stability conditions for different classes of perturbations, in section 4 we analyze the problem of robust performance, and finally in section 5 we end with a few concluding remarks.

\section{Classes of Systems and Per- turbations}

In this section we will present a precise description of the classes of systems and perturbations that are commonly used. The first two subsections describe norm-bounded classes of perturbations, and the last subsection presents real parametric uncertainty. We will also present certain classes of scaling operators that paly an important role in the stability analysis of systems with these types of perturbations. We reiterate at this point that we are not presenting the most general definitions for the sake of simplicity and readability. Comments for the general cases will be made whenever they are applicable.

\subsection{Time-Invariant Systems on $L^{p}$}

Let $L^{p}([0, \infty)]$ be the classical Banach space of scalar-valued, $p$-integrable, Lebesgue measurable functions on $[0, \infty)$. We denote by $\mathcal{B}\left(L^{p}\right)$ the space of all bounded linear operators from $L^{p}$ to $L^{p}$. The first subset of $\mathcal{B}\left(L^{p}\right)$ which will provide 
a rich class of systems and perturbations is the subset of time-invariant operators. In order to define a reasonanble and sufficiently rich subset we start from the set $\mathcal{S}$ of stable proper rational transfer functions. Any element in $\mathcal{S}$ defines a bounded operator on $L^{p}$, and thus $\mathcal{S} \subset \mathcal{B}\left(L^{p}\right)$. To enlarge this class of systems we take the closure of the set $\mathcal{S}$ with respect to the norm in $\mathcal{B}\left(L^{p}\right)$, and denote this closure by $\mathcal{S}^{p}$. Therefore $\mathcal{S}^{p}$ is the smallest complete algebra of systems that contains the finite-dimensional, stable, time-invariant systems [1]. Special cases that are frequently used in the literature are $\mathcal{S}^{1}=\mathcal{S}^{\infty}=L^{1} \oplus \delta(t)$, and $\mathcal{S}^{2} \subset \mathcal{H}^{\infty}$. Also we define $\mathcal{S}^{p}(n)$ to be the set of $n \times n$ matrices whose elements are in $\mathcal{S}^{p}$.

We will use perturbations in $\mathcal{S}^{p}$ as building blocks to obtain a rich and useful class of dynamic perturbations. We define a unit ball in $\mathcal{S}^{p}$ by

$$
\Delta_{t i}^{p}:=\left\{\Delta_{t i}^{p} \in \mathcal{S}^{p}:\left\|\Delta_{t i}^{p}\right\|_{p-i}<1\right\} .
$$

Note that the subscript $t i$ denotes timeinvariance, and $p-i$ denotes induced $p$-norm. In order to represent perturbations that occur at different locations in a feedback loop we will consider diagonal time-invariant perturbations. Therefore we define

$$
\begin{gathered}
\mathrm{D}_{t i}^{p}(n):=\left\{\operatorname{diag}\left(\Delta_{t i, 1}^{p}, \ldots, \Delta_{t i, n}^{p}\right):\right. \\
\left.\Delta_{t i, j}^{p} \in \Delta_{t i}^{p} \text { for } j=1, \ldots, n\right\} .
\end{gathered}
$$

Time invariant perturbations enjoy many interesting properties which are shown in [1], and will be summarized in the next two theorems:

Theorem 1 If $G \in \mathcal{S}^{p}$, then it follows that $G \in$ $\mathcal{S}^{q}$, where $\frac{1}{p}+\frac{1}{q}=1$. Furthermore, the two norms are equal, i.e. $\|G\|_{p-i}=\|G\|_{q-i}$.

Theorem 2 1. Let $G$ be a LTI system, then for any $1 \leq p, q \leq \infty$ with $\frac{1}{p}+\frac{1}{q}=1$, we have

$$
\|G\|_{\mathcal{H}^{\infty}}=\|G\|_{2-i} \leq\|G\|_{p-i}=\|G\|_{q-i} .
$$

2. If $2<p<\infty$, then $1<q<2$, and the following nesting relationships hold

$$
\begin{array}{ccccccccc}
\mathcal{S}^{\infty} & \subset & \cdots & \subset & \mathcal{S}^{p} & \subset & \cdots & \subset & \mathcal{S}^{2} \\
\mathcal{S}^{1} & \subset & \cdots & \subset & \mathcal{S}^{q} & \subset & \cdots & \subset & \mathcal{S}^{2}
\end{array}
$$

$$
\begin{array}{ccccccccc}
\mathbf{D}_{t i}^{\infty} & \subset & \cdots & \subset & \mathbf{D}_{t i}^{p} & \subset & \cdots & \subset & \mathbf{D}_{t i}^{2} \\
\mathbb{D}_{t i}^{1} & \subset & \cdots & \subset & \mathbb{D}_{t i}^{q} & \subset & \cdots & \subset & \mathbf{D}_{t i}^{2}
\end{array}
$$

The class of operators that commute with the classes of system perturbations play an important role in the analysis of systems with uncertainty. In considering time-invariant perturbations the following set of scalings are used:

$$
\begin{gathered}
\mathcal{T}_{t i}^{p}(n):=\left\{\operatorname{diag}\left(t_{1}(s), \ldots, t_{n}(s)\right):\right. \\
\left.t_{i}(s) \in \mathcal{S}^{p} \text { and } t_{i}^{-1}(s) \in \mathcal{S}^{p}\right\} .
\end{gathered}
$$

It should be clear that the set of scalings in $\mathcal{T}_{t i}^{p}$ commute with the elements in $D_{t i}^{p}(n)$.

\subsection{Time-Varying Perturbations}

Now we will consider a larger susbset of perturbations than $D_{t i}^{p}$, which is contained in $\mathcal{B}\left(L^{p}\right)$. Denote by $\mathcal{B}_{T V}\left(L^{p}\right)$ the elements in $\mathcal{B}\left(L^{p}\right)$ that are causal; i.e if $G \in \mathcal{B}_{T V}\left(L^{p}\right)$ then $G$ satisfies $P_{T} G P_{T}=P_{T} G$, for all $T>0$, where $P_{T}$ is the standard truncation operator. The set $\mathcal{B}_{T V}\left(L^{p}, n\right)$ is made of $n \times n$ matrices with elements in $\mathcal{B}_{T V}\left(L^{p}\right)$. Define

$$
\Delta_{t v}^{p}:=\left\{\Delta_{t v}^{p} \in \mathcal{B}_{T V}\left(L^{p}\right):\left\|\Delta_{t v}^{p}\right\|_{p-i}<1\right\} .
$$

In order to account for structured time-varying uncertainty we will use the notation

$$
\begin{gathered}
\mathbf{D}_{t v}^{p}(n):=\left\{\operatorname{diag}\left(\Delta_{t v, 1}^{p}, \ldots, \Delta_{t v, n}^{p}\right):\right. \\
\left.\Delta_{t v, j}^{p} \in \Delta_{t v}^{p}, \text { for } j=1, \ldots, n\right\} .
\end{gathered}
$$

There is no inclusion relation among the timevarying balls $\Delta_{t v}^{p}$ in an analogous way to those for $\Delta_{t i}^{p}$ which were illustrated in theorem 2 .

In studying systems with time-varying perturbations the following class of scalings play a major role

$$
\mathcal{T}_{t v}(n):=\left\{\operatorname{diag}\left(t_{1}, \ldots, t_{n}\right): t_{i} \in R, t_{i} \geq 0\right\} .
$$

\subsection{Real Parametric Uncertainty}

This class represents localized uncertainties, that can occur at any point in a feedback loop. Such perturbations are not of dynamic type, and thus cannot be easily covered by the previous types of perturbation classes. As a basic building block 


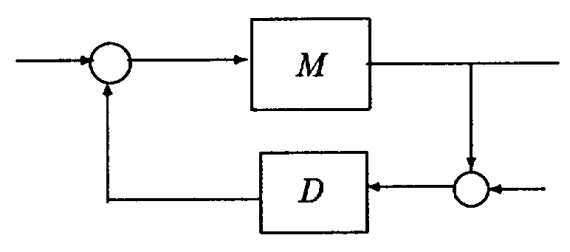

Figure 1: Robust Stability Configuration

we will use a ball in $R$ (i.e an interval) and define the following structured ball:

$$
\begin{gathered}
\mathbf{D}_{r}(n):=\left\{\operatorname{diag}\left(\Delta_{r, 1}, \ldots, \Delta_{r, n}\right):\right. \\
\left.\left|\Delta_{r, i}\right|<1, \Delta_{r, i} \in R\right\} .
\end{gathered}
$$

In this outline for the sake of simplicity we are considering only intervals in $R$, and thus there is no difference due to the $p$-norm. We will also define the perturbation class of mixed real parametric and linear time-invariant perturbations as

$$
\begin{gathered}
\mathbf{D}_{t i-r}^{p}(n, m):=\left\{\operatorname{diag}\left(D_{t i}, D_{r}\right):\right. \\
\left.D_{t i} \in \mathbf{D}_{t i}^{p}(n), D_{r} \in \mathbf{D}_{r}(m)\right\} .
\end{gathered}
$$

\section{Robust Stability Conditions}

In this section we will present conditions for robust stability of systems in the presence of different types of uncertainties. To that end consider the diagram in figure 1. The block $M$ denotes the nominal plant, which contains the original plant, the controller, and the interconnection structure of the uncertainties. The part denoted by $D$ represents the perturbation block which is made of any operator that belongs to a set of perturbations $\mathcal{P}$. We will adopt the following definition of robust stability [1]:

Definition 1 The system in figure 1 is robustly $L^{p}$-stable for a set of perturbations $\mathcal{P}$, if

(i) For all $D \in \mathcal{P},(I+M D)$ has an inverse in the appropriate algebra (i.e. in $\mathcal{S}^{p}(n)$ for the time-invariant cases, and in $\mathcal{B}_{T V}\left(L^{p}, n\right)$ for the time-varying cases).

(ii) $\sup _{D \in \mathcal{P}}\left\|(I+M D)^{-1}\right\|_{p-i}<\infty$.

\subsection{Linear Time-Invariant, and Para- metric Perturbations}

We will consider the robust stability of configuration 1 for linear time-invariant perturbations that are bounded in the $L^{p}$-induced norm sense; i.e the class $D_{t i}^{p}(n)$, as well as real parametric uncertainty. The techniques based on the structured singular value ( $\mu$-analysis) play an important role in the study of robust stability in the presence of time-invariant perturbations $[3,5]$. The structured singular value is defined on a matrix $M(j \omega)$ with respect to a class of timeinvariant perturbations $\mathcal{P}$ as folllows:

$$
\begin{gathered}
\frac{1}{\mu_{\mathcal{P}}(M(j \omega))} \\
:=\min \{\bar{\sigma}(D): \operatorname{det}(I+M D)(j \omega)=0, D \in \mathcal{P}\} .
\end{gathered}
$$

Note that $\mu$ is defined with respect to a timeinvariant perturbation class that may contain both real parametric and dynamic perturbations. Thus it can simultaneously handle both types of uncertainty, as will be illustrated later. In what follows we will suppress the subscripts on $\mu$ since the class of perturbations with respect to which $\mu$ is calculated will be clear from the context. The following theorem can be found in $[1,3]$ :

Theorem 3 Consider the interconnection in figure 1 , let $M \in \mathcal{S}^{p}(n)$ for all $p \in[1, \infty]$. For any fixed $p \in[1, \infty]$, the following statements are equivalent:

(a) System is robustly $L^{2}$-stable for the perturbation class $\mathbf{D}_{t i}^{2}(n)$.

(b) System is robustly $L^{p}$-stable for the perturbation class $\mathbf{D}_{t i}^{p}(n)$.

(c) $\sup _{\omega \in R} \mu(M(j \omega))<1$, where $\mu$ is computed with respect to the perturbation class $\mathrm{D}_{t i}^{2}(n)$.

(d) If $n \leq 3, \inf _{T \in T_{t i}^{2}(n)}\left\|T^{-1} M T\right\|_{2-i}<1$.

It is important to point out that the equivalence of the previous statements with part (b) is dependent on the assumption that the perturbation class $D_{t i}^{p}(n)$ is made of scalar perturbations. The case of multivariable perturbations will not be true due to the incompatibility of the norms defined on the finite dimensional part of the signal spaces.

The following theorem can be found in [5]: 
Theorem 4 Consider the interconnection in figure 1 , let $M \in \mathcal{S}^{2}(n+m)$. The following statements are equivalent:

(a) System is robustly $L^{2}$-stable for the perturbation class $\mathbf{D}_{t i-r}^{2}(n, m)$.

(b) $\sup _{\omega \in R} \mu(M(j \omega))<1$, where $\mu$ is computed with respect to the perturbation class $\mathrm{D}_{t i-r}^{2}(n, m)$.

\subsection{Time-Varying Perturbations}

Here we again consider the configuration in figure 1 , with the perturbation $D \in \mathrm{D}_{t v}^{p}(n)$, and $M \in \mathcal{S}^{p}(n)$. The two cases for which progress was achieved are $p=2$ and $p=\infty$ cases. The next theorem can be found in [4] and illustrates the $p=2$-case.

Theorem 5 Consider the interconnection in figure 1 , let $M \in \mathcal{S}^{2}(n)$. The following statements are equivalent:

(a) System is robustly $L^{2}$-stable for the perturbation class $\mathbf{D}_{t v}^{2}(n)$.

(b) $\inf _{T \in T_{\text {tv }}(n)}\left\|T^{-1} M T\right\|_{2-i}<1$.

The next theorem deals with the $p=\infty$-case and can be found in [2].

Theorem 6 Consider the interconnection in figure 1 , let $M \in \mathcal{S}^{\infty}(n)$. The following statements are equivalent:

(a) System is robustly $L^{\infty}$-stable for the perturbation class $\mathrm{D}_{t v}^{\infty}(n)$.

(b) $\rho(|M|)<1$, where $|M|$ is the non-negative matrix defined by $|M|_{i j}=\left\|m_{i j}\right\|_{\infty-i}$. Note that $m_{i j}$ is the $i j$-th element in $M$.

(c) $\inf _{T \in \mathcal{T}_{t v}(n)}\left\|T^{-1} M T\right\|_{\infty-i}<1$.

\section{Robust Performance}

The diagram in figure 2 illustrates the problem of robust performance. $M$ is the nominal interconnection, $w$ is an exogeneous disturbance, $z$ is the regulated output, and $D$ belongs to a perturbation class $\mathcal{P}$. The following definition is needed

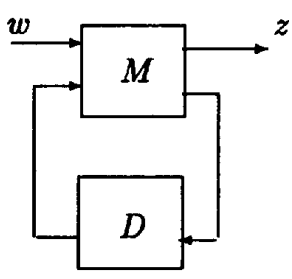

Figure 2: Robust Performance Configuration

Definition 2 The system in figure 2 achieves $L^{p}$ robust performance for a set of perturbations $\mathcal{P}$, if

(i) System is robustly $L^{p}$ stable for the set of perturbations $\mathcal{P}$.

(ii) $\sup _{D \in \mathcal{P}}\left\|T_{z w}(M, D)\right\|_{p-i}<1$, where $T_{z w}$ is the map from $w$ to $z$ in figure 2 .

Robust performance is a much harder problem to analyze than robust stability. In certain cases equivalence between robust stability and performance can be obtained which results in computational simplification. In the following theorems a robust stability problem is formed from a robust performance problem by connecting an extra perturbation between $w$ and $z$. The following theorem is based on $[1,2]$.

Theorem 7 Consider the interconnection in figure 2 , and let $M \in \mathcal{S}^{p}(n+1)$, for all $p \in[0, \infty]$. The following statements are equivalent:

(a) System achieves $L^{\infty}$-robust performance with respect to the perturbation structure $\mathrm{D}_{t i}^{\infty}(n)$.

(b) System is $L^{\infty}$-robustly stable with respect to the mixed perturbation structure $\mathbf{D}_{m}(n+1)$, which is defined as

$$
\begin{gathered}
\mathbf{D}_{m}(n+1)=\left\{\operatorname{diag}\left(D_{t v}^{\infty}, D_{t i}^{\infty}\right):\right. \\
\left.D_{t v}^{\infty} \in \mathbf{D}_{t v}^{\infty}(1), D_{t i}^{\infty} \in \mathbf{D}_{t i}^{\infty}(n)\right\} .
\end{gathered}
$$

The following theorem can be found in $[3,5]$ :

Theorem 8 Consider the interconnection in figure 2, let $M \in \mathcal{S}^{2}(n+1+s)$. The following statements are equivalent: 
(a) System achieves $L^{2}$-robust performance with respect to the perturbation structure $\mathbf{D}_{t i-r}^{2}(n, s)$.

(b) System is $L^{2}$-robustly stable with respect to the mixed perturbation structure $\mathbf{D}_{m}(1+n+$ $s)$, which is defined as

$$
\begin{gathered}
\mathbf{D}_{m}(1+n+s)=\left\{\operatorname{diag}\left(D_{t v}^{2}, D_{t i-r}^{2}\right):\right. \\
\left.D_{t v}^{2} \in \mathbf{D}_{t v}^{2}(1), D_{t i-r}^{2} \in \mathbf{D}_{t i-r}^{2}(n, s)\right\} .
\end{gathered}
$$

(c) System is $L^{2}$-robustly stable with respect to the perturbation structure $\mathbf{D}_{t i-r}^{2}(n+1, s)$.

(d) $\sup _{\omega \in R} \mu(M(j \omega))<1$, where $\mu$ is computed with respect to the perturbation structure $\mathbf{D}_{t i-r}^{2}(n+1, s)$.

The following theorem is based on [4].

Theorem 9 Consider the interconnection in figure 2, and let $M \in \mathcal{S}^{2}(n+1)$. The following statements are equivalent:

(a) System achieves $L^{2}$-robust performance with respect to the perturbation structure $\mathbf{D}_{t v}^{2}(n)$.

(b) System is $L^{2}$-robustly stable with respect to the perturbation structure $\mathrm{D}_{t v}^{2}(n+1)$.

(c) $\inf _{T \in \mathcal{T}_{i v}(n+1)}\left\|T^{-1} M T\right\|_{2-i}<1$.

The following theorem can be found in [2].

Theorem 10 Consider the interconnection in figure 2, and let $M \in \mathcal{S}^{\infty}(n+1)$. The following statements are equivalent:

(a) System achieves $L^{\infty}$-robust performance with respect to the perturbation structure $\mathrm{D}_{t v}^{\infty}(n)$.

(b) System is $L^{\infty}$-robustly stable with respect to the perturbation structure $\mathrm{D}_{t v}^{\infty}(n+1)$.

(c) $\inf _{T \in T_{t v}(n+1)}\left\|T^{-1} M T\right\|_{\infty-i}<1$.

(d) $\rho(|M|)<1$, where $|M|$ is the non-negative matrix defined in theorem 6 .

\section{Concluding Remarks}

We have briefly summarized many of the recent results dealing with robust stability and performance in the presence of mixed perturbations. For a more extensive development the reader may refer to the articles in the references.

\section{References}

[1] B. Bamieh and M. Dahleh, "On Robust Stability with Structured Time-Invariant Perturbations," to appear in Systems and Control Letters.

[2] M. A. Dahleh, and M. Khammash, "Controller Design for Plants with Structured Uncertainty," to appear in Automatica Special Issue on Robust Control.

[3] A. K. Packard, and J. C. Doyle, "The Complex Structured Singular Value," to appear in Automatica Special Issue on Robust Control.

[4] J. S. Shamma, 'Robust Stability with TimeVarying Structured Uncertainty,' to appear in 31st IEEE CDC.

[5] P. M. Young, M. P. Newlin, and J. C. Doyle, "Let's Get Real," to appear in 1992 ASME Winter Annual Meeting. 